\title{
Criteria for lung volume reduction surgery reimbursement: Too many questions, not enough answers
}

Keith S. Naunheim, MD

See related article on page 829 .
From the Department of Thoracic Surgery, St Louis University, St Louis, Mo.

Received for publication Oct 31, 2003; revisions requested Nov 3, 2003; accepted for publication Nov 20, 2003

Address for reprints: K. S. Naunheim, MD, Department of Thoracic Surgery, St Louis University, St Louis, MO 63110 (E-mail: naunheim@slu.edu).

J Thorac Cardiovasc Surg 2004;127:631-2 $0022-5223 / \$ 30.00$

Copyright $\odot 2004$ by The American Association for Thoracic Surgery

doi:10.1016/j.jtcvs.2003.11.023

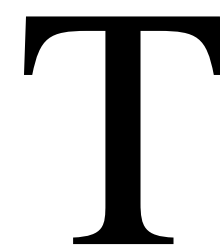

he report by Meyers and colleagues ${ }^{1}$ in this edition of the Journal regarding lung volume reduction surgery (LVRS) in high-risk patients raises some interesting methodologic and ethical questions. The article was designed to assess the validity of patient selection criteria outlined in the first clinical publication of the National Emphysema Treatment Trial (NETT). ${ }^{2}$ In that article, NETT investigators identified a high-risk patient subset characterized by the combination of a forced expiratory volume in 1 second $\left(\mathrm{FEV}_{1}\right)$ less than or equal to $20 \%$ of predicted and either a homogeneous pattern of disease or a diffusing lung capacity for carbon monoxide (DLCO) less than or equal to $20 \%$ of predicted. This subgroup experienced a high mortality and morbidity that outweighed the clinical benefit from the procedure. The report herein disputes this conclusion by demonstrating that patients with an $\mathrm{FEV}_{1}$ less than or equal to $20 \%$ and a DLCO less than or equal to $20 \%$ but who have a heterogeneous pattern of disease can benefit from LVRS. The argument goes that such patients should not be prevented from undergoing LVRS, as has been specified in the recent Medicare coverage decision, which used the NETT results as a guideline for reimbursement.

Therefore, the NETT suggests that a specific subset of patients constitute a high-risk group that should not undergo LVRS, whereas Meyers and colleagues believe that further subselection in this high-risk group can cull a subgroup with an acceptable risk-benefit ratio. Who's right?

Some readers will take this article at face value and agree with the conclusion of Meyers and colleagues on the basis of reputation alone. The investigators at Washington University have one of the largest and certainly the longest experiences with LVRS and are acknowledged leaders in the field. However, there could be unintended consequences if their criteria were universally followed. Their report documents that this patient subset, although deriving significant benefit, is certainly at high risk, with an increased chance of prolonged air leak and gastrointestinal morbidity and an incidence of reoperation 3 times as great as that quoted in their recent report of long-term results. Although it is true that their 5\% mortality is laudable, it is important to ask whether similar results can be achieved at other institutions with less experience and expertise in LVRS. The Centers for Medicare and Medicaid Services (CMS) has agreed to reimburse for LVRS at the 17 participating NETT centers and 31 non-NETT Medicare-approved lung transplant centers around the country. Whereas the NETT institutions have documented their expertise in LVRS, fewer than half of the non-NETT CMS-approved lung transplant centers have a published experience with LVRS, and many have no ongoing programs. Is it really ethical to suggest that these high-risk patients undergo LVRS at institutions with little or no experience?

Other readers will agree with the NETT perspective, believing that a prospective randomized controlled trial involving more than 1200 patients provides what are arguably the best and most definitive data available on LVRS. The trial was designed specifically to address the shortcomings, both theoretical and practical, of the early LVRS reports; these included selection bias, survivorship bias, aggregate data reporting, incomplete follow-up, and lack of medical controls. The recently published report represents the best clinical and academic efforts of 18 centers (17 clinical centers and 1 coordinating center) recognized for their expertise in the arena 
of LVR. ${ }^{3}$ Is it really reasonable to second-guess such a trial and change selection criteria on the basis of a single report?

The simplest solution would seem to be to ask the NETT investigators to review their data and sift out those 24 LVRS patients whose parameters are identical to those cited in the current report $\left(\mathrm{FEV}_{1} \leq 20 \%\right.$ of predicted, DLCO $\leq 20 \%$ of predicted, and heterogeneous disease). Analysis of this subset might provide corroborative data that reinforce the argument for reimbursement in this patient subset. However, there are significant methodologic shortcomings to this approach. The definitions for homogeneous/heterogeneous disease are not necessarily consistent between the Washington University group and the NETT. Certainly, the determination was made by different investigators with no common base of experience. There is also no standardization of methods to ensure similarity of patient selection or operative or perioperative management. Is a comparison of the results of these 2 groups really valid?

In addition, there are the statistical problems of a post hoc analysis in a retrospectively selected patient subset even if the data are prospectively collected. The original NETT data analysis was undertaken in a genuinely prospective fashion, with hypotheses and potential predictors identified before the institution of the trial. Repeated retrospective or post hoc analyses can be interpreted as data mining, a practice that is known to sometimes yield false but "statistically significant" conclusions regarding clinical outcome or patient predictors. When is such a post hoc analysis legitimate, and should it be used to change selection criteria?

This clinical dilemma is not new. Time and again over the last few decades, we have seen "definitive" results from prospective randomized trials being challenged subsequently by new and contradictory studies. This is the way the science of surgery has evolved for decades. What is new here is the active involvement by CMS in the selection of patients. Their tailoring of the reimbursement policy to coincide with the results of a single specific trial is a relatively new phenomenon in thoracic surgery. This leads to the exclusion of patients who might be good candidates for LVRS but whose specific clinical profiles were not prospectively assessed in a randomized trial. Unfortunately, this ethical dilemma is not limited to the specific high-risk subset reported by Meyers and associates. What about $\alpha_{1}$ antitrypsin patients with lower lobe-predominant heterogeneous disease? Some investigators have reported good clinical results in such patients. How about unilateral LVRS? Many reports have outlined good success after unilateral LVRS in patients with primarily unilateral disease or in those with a contraindication to operation on a single side (ie, prior thoracotomy or prior empyema). Unfortunately, published CMS guidelines prohibit reimbursement for either type of patient, and it is thus unlikely that we will be able to determine the real value of LVRS in such patients anytime soon.

The answers to all the questions cited will be neither easily obtained nor quickly forthcoming now that reimbursement is strictly constrained. It is unlikely that the CMS reimbursement guidelines will change in the near future. Unfortunately, the difficult situation we find ourselves in is likely to be repeated many times if CMS demands a "definitive" trial to prove the efficacy for each new technology and then tailors a reimbursement policy contingent on the results of that single trial. It seems probable that there will continue to be more questions than answers regarding reimbursement for new surgical technologies.

\section{References}

1. Meyers BF, Yusen RD, Guthrie TJ, Patterson GA, Lefrak SS, Davis GE, et al. Results of lung volume reduction surgery in patients meeting a National Emphysema Treatment Trial high-risk criterion. J Thorac Cardiovasc Surg. 2004;127:829-35.

2. National Emphysema Treatment Trial Research Group. Patients at high risk of death after lung volume reduction surgery. $N$ Engl J Med. 2001;345:1075-83.

3. National Emphysema Treatment Trial Research Group. A randomized trial comparing lung volume reduction surgery with medical therapy for severe emphysema. N Engl J Med. 2003;348:2059-73. 